\title{
The Use of Near Infrared Light Emitting Diode for Kienbock's Disease: A Case Study
}

\author{
Lori Burkow-Heikkinen ${ }^{1 *}$ and Erin Morency ${ }^{2}$ \\ ${ }^{1}$ Department of sports and fitnes, American College of Sports Medicine, Indianapolis, Indiana \\ ${ }^{2}$ Department of Sports Medicine, Troy Beaumont, Troy, Michigan
}

*Corresponding author: Lori Burkow-Heikkinen, Department of sports and fitnes, American College of Sports Medicine, Indianapolis, Indiana.

Received Date: July 11, 2020

Published Date: July 24, 2020

\begin{abstract}
Kienbock's disease is a relatively rare disorder where the blood supply to one of the small bones in the wrist is interrupted. The affected bone, the lunate, is one of eight small carpal bones that is in the middle at the base of the wrist and is responsible for wrist movement. This case study examines an otherwise healthy white 71-year-old female with extreme wrist pain, initially diagnosed by the provider as tiny ganglion cysts in the ulnar tissue but later appropriately diagnose Kienbock's left wrist with ulnar minus. This case study will study the care of the wrist using a 940nm near-infrared light emitting diode.
\end{abstract}

Background: There is no cure for Kienbock's disease, but prompt treatment can help preserve wrist function and relieve pain. Kienbock's disease progresses through four stages: loss of blood flow, sclerosis of bone, breakdown of lunate bone, and finally the breakdown of the remaining bones of the hand [1]. The rate of progression is varied from case to case.

Objective: To evaluate the effects of the near infrared light emitting diode on inflammation and bone healing.

Materials and Method: This article investigates the role of near infrared light emitting diode on the sclerosis of the lunate carpal bone, stage one of Kienbock's disease. A review of books, journals, PubMed, and physician notes

Discussion: Using the near infrared light emitting diode (NIR LED), healing rate has increased, a decrease in inflammation, bone regeneration, and pain has minimized. The NIR LED gallium arsenide can be used as an adjunctive therapy for reduction for this progressive disease.

Keywords: Kienbock; Osteonecrosis; Fusion; Arthroplasty; Sclerosis; Near Infrared light emitting diode; Phototherapy

\section{Introduction}

Near infrared light therapy, also known as phototherapy, is a process that tissues exhibit when exposed to light energy from sources such as lasers, light emitting diodes, and florescent lamps. During medical treatment near infrared gallium arsenide phosphate devices used for light emitting diodes (near infrared and gallium arsenide phosphate) are applied to the site of injury or pain, allowing light to directly penetrate the tissue [2]. This case study will specifically address the medical NIR LED, a monochromatic, non-coherent, solid state semi-conductor diode that emits light in the near infrared range when a current is applied through the device [3]. Gallium Arsenide (Ga-As) NIR LED therapy can be used as a coadjutant therapy to control inflammation and wound healing alongside surgery, orthopedic fixation and pharmaceutical treatments [4].

\section{Materials and Methods}

Information was gathered from literature searches in books, journals, PubMed, and Physician notes. 


\section{Result}

After regular use of the $940 \mathrm{~nm}$ NIR LED an individual saw decrease in pain and increase in mobility and function of the wrist post Kienbock's disease diagnosis. The individual was recommended to add the NIR LED therapy for 30 minutes a day to both the anterior and dorsal aspect of her wrist.

\section{Discussion}

Kienbock Disease disrupts the blood supply to the lunate, one of the small bones of the wrist, thus leading to chronic inflammation and tissue destruction. Chronic inflammation is characterized by the infiltration of mononuclear inflammatory cells, tissue destruction, and attempts at healing fibrosis and angiogenesis [5]. One of the most important growth factors in the process of angiogenesis is vascular endothelial growth factor (VEGF). Both VEGF and angiogenesis can be stimulated by near infrared light [6,7]. Newer research indicates that tumor necrosis factor (TNF)- like weak inducer of apoptosis (Tweak)/fibroblast growth factor-inducible 14 (Fn14) may help regulate various cell functions, including differentiation and proliferation. It is also believed to have a role in inflammatory conditions as well as bone metabolism. Du et al. suggest that by targeting the Tweak/Fn14 signaling pathway, and thereby osteoblast-osteoclast coupling, osteoclast resorption can be decreased, and osteoblast formation can be stimulated [8].

Many cytokines are involved in regulating the healing of a nonunion fracture, in both humans and animals. These include tumor necrosis factor- $\alpha$ (TNF- $\alpha$ ), interleukin- 6 , bone metabolic proteins, and insulin-like growth factor [9]. Nitric oxide is known to maintain vascular tone and near infrared stimulation has been observed to increase nitric oxide levels [10]. Nitric oxide synthase 2(NOS2) and IL-12B gene expression are both regulated by mechanical shear and compression. Inflammatory proteins increase when mechanical stimulation is introduced [11]. Bone remodeling is affected by these inflammatory factors along with changes in mechanical stress [12].

By interrupting endothelial signaling Ramasamy et al. observed decreased osteogenesis and bone mass in mice. This led to the conclusion that there is a correlation between angiogenesis and osteogenesis [13]. Osteoblast and osteoclast activity is influenced by cytokines that are produced by osteocytes, which make up the greatest portion of cells found in bone. Two of the cytokines that are important at stimulating bone healing are Interleukin (IL)6 and IL-8 [14]. IL-10, integrin avB3, and nuclear factor kappa B are all affected by osteopontin, which in turn helps to control osteoclast activity [15]. Other modulators that play active roles in bone regeneration after a fracture include $\mathrm{T}$ lymphocytes and IL17F [16].

Just like bone repair, growth factors and cytokines play a mandatory role in muscle repair. Near infrared light emitting diode phototherapy decreases IL-1B, thereby playing a role in the acute inflammatory response and stimulate skeletal muscle repair [17]. Endothelial cells of blood vessels serve many functions, including regulating developmental and regenerative growth of bone [18].

One form of treatment for bone regeneration includes autogenous bone grafts. With the addition of laser photo biomodulation, in the form of a low-level laser or LED, marked bone regeneration, integration, and decreased inflammation have been observed [19]. In the article lead by Pinheiro, beneficial outcomes were observed with the use of either a laser or LED by increasing collagen matrix creation [20].

In the study by Tani et. al, they stated different areas of medicine have had positive outcomes with the addition of photo biomodulation to treatment. They concluded that the use of a $635 \mathrm{~nm}$ laser had potentially beneficial results for improving bone regeneration [21]. In another study by Zein et al, they also concluded that there is a positive correlation between the use of a low-level laser and bone regeneration. They did note that there seemed to be a relationship between dose and power. They believe the positive effects are because of increased cellular metabolism, and protein synthesis, resulting in the increased bone regeneration [22].

Kienbock's Disease is a rare disorder that disrupts the blood supply to the lunate bone. The lunate bone is one of the small bones in the wrist which is important for wrist function. When the blood supply to the bone stops the bone dies, called osteonecrosis. Kienbock's disease progresses through four stages, the time spent in any one stage varies for every individual. Depending on the degree of damage there are several surgical options available: Revascularization, capitates-shortening osteotomy, joint leveling, metaphyseal core decompression, proximal row carpectomy, fusion, and arthroplasty [23-25].

This case study involves a 71-year-old Caucasian female. The patient started showing symptoms of wrist pain, beginning in the summer of 2018, which progressed for six months prior to her seeking diagnosis. The patient felt better having the wrist supported with a neoprene wrist support, however it was pulled so tight that she started to develop atrophy of the fingers, wrist, and forearm.

When first seen, at the beginning of 2019, radiology showed diffuse edema signal with areas of sclerosis within the lunate, compatible with the acute phase of osteonecrosis, associated joint effusion. Scattered degenerative cystic and erosive changes throughout the carpal bones. Also present were tiny ganglion cysts in the ulnar aspect of the wrist and hand.

\section{Technique: MR imaging of left wrist without contrast.}

Interpretation: Osseous structure: degenerative cystic changes of the trapezium at the ulnar aspect.

Complete signal abnormality of the lunate with edema signal mixed with the areas of sclerosis. Most likely represents the acute 
phase of osteonecrosis.

Musculature: The visualized musculature demonstrates normal signal.

Fluid: There is small wrist joint effusion.

Tendons: The visualized tendons are intact with normal signal and morphology.

Carpal Tunnel and Guyon's canal: The contents of the carpal tunnel and Guyon's canal are within normal limits. 3mm ganglion cysts at the ulnar aspect of the wrist at the level of ulnar styloid and also at the level of the fifth carpometacarpal joint, immediately adjacent to the bone. Three weeks after the first MRI patient was given the near infrared light emitting diode (NIR LED), a $940 \mathrm{~nm}$ device, and was told to use the device for 30 minutes on the dorsal aspect of the wrist and 30 minutes on the anterior aspect of the wrist (Figure 1\&2).

A second evaluation by a different specialist revealed no evidence of a cyst and was diagnosed with Kienbock's, along with the recommendation of surgical intervention. The second radiology report was as follows (see images 1 and 2):

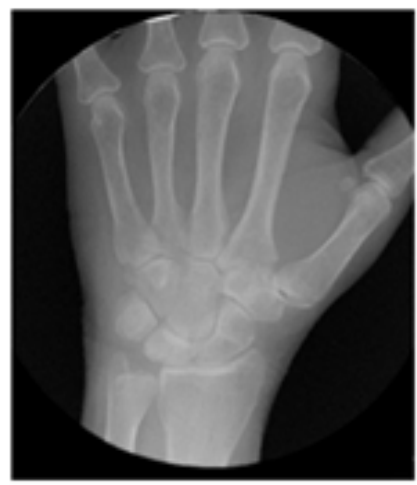

Figure 1: Left wrist.

Figure 1: Left wrist.

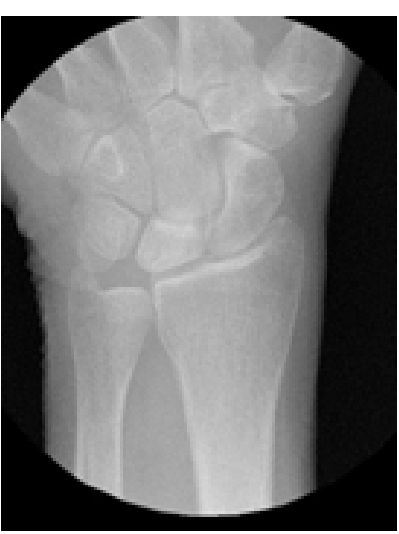

History: wrist pain.

Examination: Three views of the left wrist.

Findings: Negative for fracture or dislocation. Soft tissues are intact. Osseous mineralization is normal.

PA view: The radius and ulna are intact. There is no intercarpal gap.

There is sclerosis of the left lunate. The left lunate shows a linear fracture and indentation at the proximal articular surface of the lunate at the radial lunate joint, present midway in the lunate from radial to ulnar. On the left there is ulnar $-2 \mathrm{~mm}$. The scapholunate angle is $55^{*}$ on the left carpal height index is 0.5 .

Lateral view: The lunate is neutral. The DRUJ is intact.
Oblique view: No bony mass or cystic change.

Impression: Kienbock's left wrist with ulnar minus.

The patient was told to remove the neoprene brace whenever possible to help reduce atrophy and put on anti-inflammatory medication. The patient followed recommendations as well as continued using the near infrared light emitting device. At a four month follow up visit on visual examine, the patient regained muscle strength as well as decreased pain. A further X-ray has been postponed for later.

\section{Conclusion}

Near Infrared light emitting diode therapy has been shown to have beneficial gains when used along with more traditional forms 
of therapy, physical therapy, chemical treatment, surgical treatment etc. In this case study the patient's lunate bone seemed to heal with the use of the $940 \mathrm{~nm}$ NIR LED on the site of injury. The patient reported better mobility and flexibility, as well as decreased pain. The patient reported greater ability to complete activities of daily living after a course of treatment involving the NIR LED. Further case studies and double-blind studies should be conducted to better determine the potential benefits of coadjutant NIR LED therapy on the early stage of Kienbock's Disease.

\section{Acknowledgement}

None.

\section{Conflicts of Interest}

No Conflicts of Interest.

\section{References}

1. Allan CH, Joshi A, Lichtman DM (2001) Kienbock's disease: diagnosis and treatment. J Am Acad Orthop Surg 9(2): 128-136.

2. Jagdeo J, Adams L, Brody N, Seigel D (2012) Transcranial red and near infrared light transmission in cavdaveric model. PloSONE 7e47460.

3. Burkow L, Stockwell N, Morency E, Sosa P (2014) The use of near infrared light emitting diode in treating sports-related injuries: a review. Research 1: 1278.

4. Whelan HT, Smits RL, Buchman EV, Whelan NT, Turner SG, et al. (2001) Effect of NASA Light-emitting diode irradiation on wound healing. J ClinLaser Med Surg 19(6): 305-314.

5. Blum A, Zarqh O, Peleg A, Sirchan R, Blum N, et al. (2012) Vascular inflammation and endothelial dysfunction in fracture healing. Am J Orthop 41(2): 87-91.

6. Salate AC, Barbosa G, Gasper P, Koeko PU, Paizotto Na, et al. (2005) Effect of In-Ga-Al-P diode laser irradiation on angiogenesis in patial ruptures of Achilles tendon in rats. Photomed Laser Surg 23(5): 470-475.

7. Liu Y, Wang D, Wu X, Zhou J (2019) Ischemia Injury: A New method accelerates bone healing in a rat tibia fracture model. BioMed Research International (4): 1-10.

8. Du YY, Zhao YX, Liu YP, LIU W, Wang MM, et al. (2015) Regulatory Tweak/Fn14 signaling pathways as a potent target for controlling bone loss. Biomed Pharmacother $70: 170-173$.

9. Ding ZC, Lin YK, Gan YK, Tang TT (2018) Molecular pathogenesis of fracture nonunion. J Orthop Translat 14: 45-56.

10. Mitchell UH, Mack GL (2013) Low-level laser treatment with near infrared light increases venous nitric oxide levels acutely; a single-blind, randomized clinical trial efficacy. Am J Phys Med Rehabil 92(2): 151-156.

11. Fahy N, Menzel U, Alini M, Stoddart MJ (2019) Shear and dynamic compression modulates the inflammatory phenotype of human monocytes in vitro. Front Immunol 10: 383.
12. Metzger CE, Gong S, Aceves M, Bloomfield SA, Hook MA (2019) Osteocytes reflect a pro-inflammatory state following spinal cord injuryin rodent model. Bone 120: 465-475.

13. Ramasamy SK, Kusumbe AP, WangL, Adams RH (2014) Endothelial notch activity promotes angiogenesis and osteogenesis in bone. Nature 507(7492): 376-380.

14. Wu X, Ma Y, Chen H, Hao Z, Su N, et al. (2019) Lysophosphatidic acid induces interleukin- 6 and CXCL15 secretion from MLO-Y4 cells through activation of the LPA1 receptor and PKC $\theta$ signaling pathway. Int Immunopharmacol 70: 1405664.

15. Icer MA, Gezmen-Karadag M (2018) The multiple functions and mechanisms of osteopontin. Clin Biochem 59: 17-24.

16. Wang Y, Kim J, Chan A, Whyne C, Nam D (2018) A two-phase regulation of bone regeneration: IL-17F mediates osteoblastogenesis via C/EBP-B in vitro. Bone 116: 47-57.

17. Fernandes KP, Alves AN, Nunes FD, Souza NH, Silva JA, et al. (2013) Effect of photobiomodulation on expression of IL-1B in skeletal muscle following acute injury. Laser Med Sci 28(3): 1043-1046.

18. Watson EC, Adams RH (2018) Biology of bone: The vasculature of the skeletal system. Cold Spring Harb Perspect Med 8(7).

19. De Oliveira Goncalves JB, Buchaim DV, de Souza Bueno CR, Pomini KT, Barraviera B, et al. (2016) Effects of low-level laser therapy on autogenous bone graft stabilized with a new heterologous fibrin sealant. J Photochem Photobiol B 162: 663-668.

20. Pinheiro ALB, Soares LGP, Da Silva ACP, Santos NRS, Da Silva APLT, et al. (2018) Laser/LED phototherapy on the repair of tibial fracture treated with wire osteosynthesis evaluated by Raman spectroscopy. Laser Med Sci 33(8): 1657-1666

21. Tani A, Chellini F, Giannelli M, Nosi D, Zecchi-Orlandini S, et al. (2018) Near-Infrared $(808 \mathrm{~nm})$, and Violet-Blue $(405 \mathrm{~nm})$ photobiomodulation potentially on human osteoblasts and mesenchymal stromal cells: a morphological and molecular in vitro study. Int J Mol Sci 19(7).

22. Zein R, Selting W, Benedicenti S (2017) Effect of low-level laser therapy on bone regeneration during osseointegration and bone graft. Photomed Laser Surg 35(12): 649-658.

23. Frerix M, Kroger K, Szalay G, Muller-Ladner U, Tarner IH (2016) Is osteonecrosis of the lunate bone underestimated feature of systemic sclerosis? A case series of nine patients and review of literature. Semin Arthritis Rheum 45(4): 446-454

24. Hong IT, Lee S, Jang GC, Kim G, Han SH (2019) Kienbock's disease with non-negative ulnar variance: Treatment with combines radial wedge and shortening osteotomy. Orthopade 48(1): 96-101.

25. Kim S, Eichenauer F, Asmus A Mutze S, Eisenschenk A, Honigmann $P$ (2019) Superselective angiography of the wrist in patients with Kienbock disease. BMC Musculoskelet Disord 20(1): 143 